\title{
A reduced order extrapolating technique of solution coefficient vectors to collocation spectral method for telegraph equation
}

\author{
Hui Wang ${ }^{1}$, Qingfang He ${ }^{1}$ and Zhendong LuO ${ }^{2^{*}}$ (D)
}

\section{"Correspondence: zhdluo@163.com ${ }^{2} \mathrm{School}$ of Mathematics and Physics, North China Electric Power University, Beijing, China Full list of author information is available at the end of the article}

\begin{abstract}
In this article, we mainly develop a reduced order extrapolating model for the solution coefficient vectors of the classical collocation spectral (CCS) scheme to the two-dimensional (2D) telegraph equation by means of a proper orthogonal decomposition (POD). Therefore, we first present the CCS scheme, offer the existence, stability, and error estimates to the SC solutions, and rewrite the CCS scheme into a matrix-form. We then build a reduced order extrapolating collocation spectral (ROECS) model and analyze the existence and stability as well as errors of the ROECS solutions by some matrix tools. We finally verify the reliability and validity of the ROECS model by means of two sets of numerical simulations for the magnetic field produced by two parallel wires with the same voltage.
\end{abstract}

MSC: $65 \mathrm{~N} 35 ; 65 \mathrm{~N} 12 ; 65 \mathrm{M} 15$

Keywords: Reduced order extrapolating technique; Proper orthogonal decomposition; Telegraph equation; Stability and convergence

\section{Introduction}

Let $\Omega \subset \mathbb{R}^{2}$ be a bounded region with boundary $\partial \Omega$. We are concerned with the following telegraph equation:

$$
\left\{\begin{array}{l}
u_{t t}-\Delta u+\alpha u_{t}+\beta u=f(t, x, y), \quad t \in(0, T],(x, y) \in \Omega, \\
u(t, x, y)=0, \quad t \in(0, T],(x, y) \in \partial \Omega, \\
u(0, x, y)=G_{0}(x, y), \quad u_{t}(0, x, y)=G_{1}(x, y), \quad(x, y) \in \Omega,
\end{array}\right.
$$

where $u$ represents the unknown voltage or current, $u_{t}=\partial u / \partial t, u_{t t}=\partial^{2} u / \partial t^{2}, \Delta=\partial^{2} / \partial x^{2}+$ $\partial^{2} / \partial y^{2}, \alpha=G R(L \hat{C})^{-1}, \beta=(R \hat{C}+G L)(L \hat{C})^{-1}$, while $G$ is the known dielectric material conductance, $R$ stands for the known conductor distributing resistance, $L$ represents the known distributing inductance, $\hat{C}$ represents the known capacitance between two conductors, $T$ stands for the ultima time, and $G_{0}(x, y), G_{1}(x, y)$, and $f(t, x, y)$ are three known functions.

(c) The Author(s) 2020. This article is licensed under a Creative Commons Attribution 4.0 International License, which permits use sharing, adaptation, distribution and reproduction in any medium or format, as long as you give appropriate credit to the original author(s) and the source, provide a link to the Creative Commons licence, and indicate if changes were made. The images or other third party material in this article are included in the article's Creative Commons licence, unless indicated otherwise in a credit line to the material. If material is not included in the article's Creative Commons licence and your intended use is not permitted by statutory regulation or exceeds the permitted use, you will need to obtain permission directly from the copyright holder. To view a copy of this licence, visit http://creativecommons.org/licenses/by/4.0/. 
The telegraph equation possesses very significant physical meanings. It can be used for simulating the electric signal propagation in transmission cable and the interaction between diffusion and reaction in biology and physics branches (see [1, 2]). But the telegraph equation in real world usually contains the complex initial and boundary values, or source function, or discontinuous coefficients. Thus, it has generally no genuine solution, one has to rely on numerical solutions.

The accuracy of spectral method (see [1-11]) is far higher than that of the finite element (FE), finite difference (FD), and finite volume element (FVE) methods (see [12-18]) as its unknown functions are approximated with the smoothing functions like triangle functions or Legendre's, Jacobi's, and Chebyshev's polynomials, while the unknown functions for the FE and FVE models are approximated with the general polynomials, but the derivatives of the unknown functions of the FD method are approximated via difference quotients. In particular, the CCS model for the telegraph equation in [18] possesses the super-convergence with respect to spatial variables, but it includes lots unknowns. Therefore, the round off errors in the calculations are accumulated very rapidly, resulting in floating point overflow after computing some steps and being unable to obtain desired results. Hence, the issue of how to reduce the unknowns of the CCS format to retard the round off error amassing is urgent, and it needs to be solved in practical applications (such as mechanic engineering), which is the main objective of this paper.

Many numerical experimentations (see, e.g., [19-29]) have verified that the POD technique can immensely lessen the unknowns in the numerical methods and retard the round off error accumulation and the calculating load. It is successfully used for reduced order in the Galerkin, FE, FD, and FVE methods as well as the parametric problems as just mentioned.

Unfortunately, as we are concerned, so far there have been no reports on the ROECS model for the telegraph equation based on POD. Hance, we here set up an ROECS model of matrix-form for the coefficient vectors of the CCS solutions such that the ROECS model possesses the same base functions as the CCS one and is simultaneously equipped with merits that the CCS model possesses the higher accuracy and the POD method could lessen the unknowns. In addition, we employ the matrix theory to demonstrate the existence, convergence, and stability for the ROECS solutions such that the theoretical argumentation becomes very succinct. In so doing, the ROECS model totally distinguishes from the existing POD-based reduced order ones as stated in the above-mentioned.

The paper is organized as follows. In Sect. 2, the CCS method to the telegraph equation is proposed. Based on the proposal, in Sect. 3, we make up snapshot matrix with the first few CCS solution coefficient vectors, producing a series of POD bases from the snapshot matrix, building the ROECS format of matrix-form via the POD bases, and proving the existence, convergence, and stability to the ROECS solutions via the matrix theory. Section 4 supplies two sets of numeric experimentations in the magnetic field produced by two parallel wires with the same voltage to check out that the numeric computational results are consistent with the theory consequences and the ROECS format is very efficient when solving the telegraph equation. Section 5 summarizes the main conclusions of this study.

\section{The CCS method of the telegraph equation}

\subsection{The CCS method}

Since the closed bounded region $\bar{\Omega}$ is able to be approximately covered by several rectangles $\left[a_{i}, b_{i}\right] \times\left[c_{i}, d_{i}\right](1 \leq i \leq I)$ and by formulas $x^{\prime}=2\left(x-a_{i}\right) /\left(b_{i}-a_{i}\right)-1$ and $y^{\prime}=$ 
$2\left(y-c_{i}\right) /\left(d_{i}-c_{i}\right)-1$ we can insure $\left[a_{i}, b_{i}\right] \leftrightarrow[-1,1]$ and $\left[c_{i}, d_{i}\right] \leftrightarrow[-1,1]$, respectively, assuming that $\bar{\Omega}=[-1,1] \times[-1,1]$, i.e., $\Omega=(-1,1) \times(-1,1)$.

Let $P_{N}$ be an interpolation subspace. For convenience, let $\left\{\omega_{k}\right\}_{k=0}^{N}$ be a set of weights and $\left\{y_{k}\right\}_{k=0}^{N}$ and $\left\{x_{k}\right\}_{k=0}^{N}$ be, respectively, two groups of Chebyshev-Gauss-Lobatto (CGL) quadrature points in the $y$ and $x$ directions (see [4]), which hold the same number and are denoted by

$$
y_{k}=-\cos \frac{\pi k}{N}, \quad x_{k}=-\cos \frac{k \pi}{N}, \quad \omega_{k}=\frac{\pi}{c_{k} N}, \quad 0 \leq k \leq N
$$

the above $c_{0}=c_{N}=2$ and $c_{k}=1(1 \leq k \leq N-1) .\left\{y_{j}\right\}_{j=0}^{N}$ and $\left\{x_{k}\right\}_{k=0}^{N}$ as well as $\left\{\omega_{k}\right\}_{k=0}^{N}$ have the following properties (see [4, p. 44]).

Theorem 1 The sets of CGL quadrature points $\left\{x_{k}\right\}_{k=0}^{N}$ and $\left\{y_{k}\right\}_{k=0}^{N}$, and the sets of weights $\left\{\omega_{k}\right\}_{k=0}^{N}$ satisfy

$$
\int_{-1}^{1} \int_{-1}^{1} \omega(x, y) q(x, y) \mathrm{d} x \mathrm{~d} y=\sum_{j=0}^{N} \sum_{k=0}^{N} q\left(x_{j}, y_{k}\right) \omega_{j} \omega_{k}, \quad \forall q(x, y) \in P_{2 N-1},
$$

where $\omega(x, y)=\omega(x) \omega(y), \omega(x)=1 / \sqrt{1-x^{2}}$, and $\omega(y)=1 / \sqrt{1-y^{2}}$.

In fact, the CCS method aims to seek an approximated solution of $u(x, y)$ via the following formula:

$$
u_{N}(x, y)=\sum_{j=0}^{N} \sum_{k=0}^{N} u_{N}\left(x_{j}, y_{k}\right) h_{j}(x) h_{k}(y)=\boldsymbol{U}_{N} \cdot \boldsymbol{H}(x, y), \quad u_{N}(x, y) \in P_{N}
$$

in which $h_{j}(x)$ and $h_{k}(y)$ are Lagrange's interpolated multinomials of the CGL quadrature points, $\boldsymbol{U}_{N}=\left(u_{N_{0,0}}, u_{N_{1,0}}, \ldots, u_{N_{N, 0}}, u_{N_{0,1}}, u_{N_{1,1}}, \ldots, u_{N_{N, 1}}, \ldots, u_{N_{0, N}}, \ldots, u_{N_{N, N}}\right)^{T}$, and $\boldsymbol{H}(x, y)=$ $\left(h_{0}(x) h_{0}(y), h_{1}(x) h_{0}(y), \ldots, h_{N}(x) h_{0}(y), h_{0}(x) h_{1}(y), h_{1}(x) h_{1}(y), \ldots, h_{N}(x) h_{1}(y), \ldots, h_{0}(x) h_{N}(y)\right.$, $\left.h_{1}(x) h_{N}(y), \ldots, h_{N}(x) h_{N}(y)\right)^{T}$. Furthermore, the derivatives of $u_{N}(x, y)$ for $x$ at $x_{k}$ are formulated with

$$
\frac{\partial u_{N}\left(x_{k}, y\right)}{\partial x}=\sum_{j=0}^{N} \sum_{l=0}^{N} u_{N}\left(x_{j}, y_{l}\right) h_{j}^{\prime}\left(x_{k}\right) h_{l}(y)=\boldsymbol{U}_{N} \cdot \frac{\partial}{\partial x} \boldsymbol{H}\left(x_{k}, y\right), \quad 0 \leq k \leq N,
$$

where

$$
h_{i}^{\prime}\left(x_{k}\right)= \begin{cases}-\frac{2 N^{2}+1}{6}, & i=k=0, \\ \frac{c_{k}}{c_{i}} \frac{(-1)^{k+i}}{x_{k}-x_{i}}, & i \neq k, 0 \leq i, k \leq N, \\ -\frac{x_{k}}{2\left(1-x_{k}^{2}\right)}, & 1 \leq i=k \leq N-1, \\ \frac{2 N^{2}+1}{6}, & i=k=N .\end{cases}
$$

In the above formulas, it should be noted $c_{0}=c_{N}=2$ and $c_{k}=1(1 \leq k \leq N-1)$. By replacing $x$ with $y$ in (5) and (4), one immediately gains the calculating formulas for $\partial u_{N}\left(x, y_{k}\right) / \partial y$. 


\subsection{Some useful Sobolev spaces}

The Sobolev spaces and their norms arisen in the subsequent are classical (see [30,31]). For instance, $L^{2}(\Omega)$ denotes a set of square-integrable functions in $\Omega$ that endows the norms as well as inner product as follows:

$$
\|v\|_{0}=\left(\int_{\Omega}|v|^{2} \mathrm{~d} x \mathrm{~d} y\right)^{1 / 2}, \quad(u, v)=\int_{\Omega} u v \mathrm{~d} x \mathrm{~d} y, \quad \forall u, v \in L^{2}(\Omega) .
$$

For a dual-index $\boldsymbol{\alpha}=\left(\alpha_{1}, \alpha_{2}\right)$ (here integers $\left.\alpha_{i} \geq 0\right)$ and an integer $m \geq 0$, the norm and semi-norm in $H^{m}(\Omega)=\left\{v \in L^{2}(\Omega): D^{\alpha} v \in L^{2}(\Omega), 0 \leq|\alpha| \leq m\right\}$ are defined as

$$
\|v\|_{m}=\left(\sum_{|\alpha|=0}^{m}\left\|D^{\alpha} v\right\|_{0}^{2}\right)^{1 / 2}, \quad|v|_{m}=\left(\sum_{|\alpha|=m}\left\|D^{\alpha} v\right\|_{0}^{2}\right)^{1 / 2}, \quad \forall v \in H^{m}(\Omega),
$$

where $D^{\alpha} v=\frac{\partial^{\alpha_{1}+\alpha_{2} v}}{\partial x^{\alpha_{1}} \partial y^{\alpha_{2}}}$.

Additionally, let $\omega=1 / \sqrt{\left(1-x^{2}\right)\left(1-y^{2}\right)}$, let $\Omega=(-1,1)^{2}$, let $L_{\omega}^{2}(\Omega)$ stand for the set that all functions are square-integrable in $\Omega$ about $\omega$, which is, respectively, equipped with the norm inner product as follows:

$$
\|u\|_{0, \omega}=\left(\int_{\Omega}|u|^{2} \omega \mathrm{d} x \mathrm{~d} y\right)^{1 / 2}, \quad(u, v)_{\omega}=\int_{\Omega} u v \omega \mathrm{d} x \mathrm{~d} y, \quad \forall u, v \in L^{2}(\Omega),
$$

and let $H_{\omega}^{m}(\Omega)=\left\{v \in L_{\omega}^{2}(\Omega): D^{\alpha} v \in L_{\omega}^{2}(\Omega), 0 \leq|\alpha| \leq m\right\}$ stand for a weighted Sobolev space in $\Omega$ about $\omega$, which is equipped with a norm

$$
\|u\|_{m, \omega}=\left(\sum_{|\alpha|=0}^{m}\left\|D^{\alpha} u\right\|_{0, \omega}^{2}\right)^{\frac{1}{2}} .
$$

Moreover, let $H_{0, \omega}^{1}(\Omega)=\left\{u \in H_{\omega}^{1}(\Omega):\left.u\right|_{\partial \Omega}=0\right\}$, let $L_{\omega}^{2}(\Omega)=H_{\omega}^{0}(\Omega)$, and let

$$
H^{l}\left(0, T ; H_{\omega}^{m}(\Omega)\right) \equiv\left\{v(t) \in H_{\omega}^{m}(\Omega):\|v\|_{H^{l}\left(H_{\omega}^{m}\right)}^{2}<\infty\right\}
$$

where $\|v\|_{H^{l}\left(H_{\omega}^{m}\right)}^{2}=\int_{0}^{T} \sum_{i=0}^{l}\left\|\mathrm{~d}^{i} v(t) / \mathrm{d} t^{i}\right\|_{m, \omega}^{2} \mathrm{~d} t$.

In addition, define the $H_{\omega}^{1}$-orthogonal projection $R_{N}: H_{0, \omega}^{1}(\Omega) \rightarrow P_{N}$ such that, for any $u \in H_{0, \omega}^{1}(\Omega)$, there holds

$$
\left(\nabla\left(R_{N} u-u\right), \nabla v_{N}\right)_{\omega}=0, \quad \forall v_{N} \in P_{N}
$$

equivalently,

$$
R_{N} u(x, y)=\sum_{j=0}^{N} \sum_{k=0}^{N} R_{N} u\left(x_{j}, y_{k}\right) h_{j}(x) h_{k}(y)
$$

where $R_{N} u\left(x_{j}, y_{k}\right)$ s are values of the solution $R_{N} u(x, y)$ of (6) at points $\left(x_{j}, y_{k}\right)$.

Thus, a function $u(x, y)$ may be approximated with $R_{N} u(x, y)$, too. Furthermore, $R_{N}$ possesses the following properties (see [4, Theorems 2.16-2.18]). 
Theorem $2 \forall w \in H_{\omega}^{k}(\Omega)(k \geq 1)$ satisfies

$$
\left\|\nabla R_{N} w\right\|_{0, \omega} \leq\|\nabla w\|_{0, \omega} ; \quad\left\|\partial^{m}\left(R_{N} w-w\right)\right\|_{0, \omega}=O\left(N^{m-k}\right), \quad 0 \leq m \leq k \leq N+1
$$

\subsection{The CCS method of the telegraph equation}

Consider the following variational form of the telegraph equation.

Problem 1 Seek $u \in H_{0, \omega}^{1}(\Omega)$ such that, $\forall t \in(0, T)$,

$$
\left\{\begin{array}{l}
\left(u_{t t}+\alpha u_{t}+\beta u, v\right)_{\omega}+(\nabla u, \nabla v)_{\omega}=(f, v)_{\omega}, \quad \forall v \in H_{0, \omega}^{1}(\Omega), \\
u(0, x, y)=G_{0}(x, y), \quad u_{t}(0, x, y)=G_{1}(x, y), \quad(x, y) \in \Omega .
\end{array}\right.
$$

The next consequences of the existence as well as the stability for the solution to Problem 1 have been given in [18, Theorem 4].

Theorem 3 When $f \in L^{2}\left(0, T ; L_{\omega}^{2}(\Omega)\right)$ and $G_{1} \in L_{\omega}^{2}(\Omega)$ as well as $G_{0} \in H_{\omega}^{1}(\Omega)$, Problem 1 has a unique solution satisfying the following stability:

$$
\|u\|_{1, \omega}+\left\|u_{t}\right\|_{0, \omega} \leq \tilde{\sigma}\left(\|f\|_{L^{2}\left(H_{\omega}^{-1}\right)}+\left\|G_{1}\right\|_{0, \omega}+\left\|G_{0}\right\|_{1, \omega}\right)
$$

where $\tilde{\sigma}=2 \sqrt{\max \left\{\beta, 1,0.5 \alpha^{-1}\right\} / \min \{1, \beta\}}$.

In order to settle Problem 1 with the CCS method, one needs to discretize $u_{t t}$ and $u_{t}$ with the second order time difference quotients and the spatial variables with the CCS technique. The main aim for the CCS method is to seek all approximate solutions at the CGL quadrature points and at time nodes $t_{n}=n \Delta t$ (where $T / K=: \Delta t$ is the time step and $K>0$ is integer meeting $T=K \Delta t)$ such that $u(x, y, n \Delta t), u_{t}, u_{t t}$, and $u^{n}(x, y)$ are respectively approximated with $u^{n},\left(u^{n+1}-u^{n-1}\right) /(2 \Delta t),\left(u^{n+1}-2 u^{n}+u^{n-1}\right) / \Delta t^{2}$, and $u_{N}^{n}(x, y)$, namely

$$
u^{n}(x, y) \approx u_{N}^{n}(x, y)=\sum_{j=0}^{N} \sum_{k=0}^{N} u_{N}^{n}\left(x_{j}, y_{k}\right) h_{k}(y) h_{j}(x), \quad 0 \leq n \leq K .
$$

Then the CCS model of the telegraph equation can be established as follows.

Problem 2 Seek $u_{N}^{n} \in U_{N} \equiv H_{0, \omega}^{1}(\Omega) \cap P_{N}$ such that

$$
\left\{\begin{array}{l}
\left(u_{N}^{n+1}-2 u_{N}^{n}+u_{N}^{n-1}, v_{N}\right)_{\omega}+\frac{\Delta t^{2}}{2}\left(\nabla u_{N}^{n+1}+\nabla u_{N}^{n-1}, \nabla v_{N}\right)_{\omega} \\
\quad+\frac{\alpha \Delta t}{2}\left(u_{N}^{n+1}-u_{N}^{n-1}, v_{N}\right)_{\omega}+\frac{\beta \Delta t^{2}}{2}\left(u_{N}^{n+1}+u_{N}^{n-1}, v_{N}\right)_{\omega} \\
=\Delta t^{2}\left(f\left(x, y, t_{n}\right), v_{N}\right)_{\omega}, \quad \forall v_{N} \in U_{N}, 1 \leq n \leq K-1, \\
u_{N}^{0}(x, y)=R_{N} G_{0}(x, y), \quad u_{N}^{1}(x, y)=2 \Delta t R_{N} G_{1}(x, y)+u_{N}^{0}, \quad(x, y) \in \Omega .
\end{array}\right.
$$

The next consequences of the existence and convergence as well as stability for the CCS solutions of Problem 2 had been proven in [18, Theorems 6 and 8]. 
Theorem 4 When $f \in L^{2}\left(0, T ; L_{\omega}^{2}(\Omega)\right)$ and $G \in H_{\omega}^{1}(\Omega)$ as well as $H \in H_{\omega}^{1}(\Omega)$, Problem 2 has a unique series of solutions $u_{N}^{n} \in U_{N}(n=1,2, \ldots, K)$ that satisfy the following stability:

$$
\begin{aligned}
\left\|u_{N}^{n}\right\|_{1, \omega} \leq & \left(\frac{8+C_{p}^{2}+\beta}{C_{p}^{2} \min \{1, \beta\}}\right)^{1 / 2}\left(\left\|\nabla G_{0}\right\|_{0, \omega}+\left\|\nabla G_{1}\right\|_{0, \omega}\right) \\
& +\left(\frac{\Delta t}{\alpha \min \{1, \beta\}} \sum_{j=1}^{n}\left\|f\left(t_{j}\right)\right\|_{0, \omega}^{2}\right)^{1 / 2}, \quad n=1,2, \ldots, K .
\end{aligned}
$$

In addition, if the solutions $u\left(t_{n}\right) \in H_{\omega}^{m}(\Omega)(2 \leq m \leq N+1)$ to Problem 1 , the error estimates between the solution of Problem 1 and the solutions of Problem 2 are as follows:

$$
\left\|u\left(t_{n}\right)-u_{N}^{n}\right\|_{1, \omega}=O\left(\Delta t^{2}, N^{-m}\right), \quad 2 \leq m \leq N+1,1 \leq n \leq K .
$$

\subsection{The matrix-form of the CCS model}

In the following, we rewrite the CCS model as the matrix-form. To do this, let

$$
u_{N}^{n}=\sum_{k=0}^{N} \sum_{i=0}^{N} u_{N_{k, i}}^{n} h_{k}(y) h_{i}(x) .
$$

By taking $v_{N}=h_{m}(y) h_{l}(x) \in U_{N}(0 \leq l, m \leq N)$ in Problem 2, we gain the formulas

$$
\begin{aligned}
& \left(u_{N}^{n+1}, v_{N}\right)_{\omega}=\sum_{k=0}^{N} \sum_{i=0}^{N} u_{N_{k, i}}^{n+1}\left(h_{k}(y) h_{i}(x), h_{m}(y) h_{l}(x)\right)_{\omega} \\
& \left(\nabla u_{N}^{n+1}, \nabla v_{N}\right)_{\omega}=\sum_{k=0}^{N} \sum_{i=0}^{N} u_{N_{k, i}}^{n+1}\left[\left(h_{k}(y) h_{i}^{\prime}(x), h_{m}(y) h_{l}^{\prime}(x)\right)_{\omega}+\left(h_{k}^{\prime}(y) h_{i}(x), h_{m}^{\prime}(y) h_{l}(x)\right)_{\omega}\right] .
\end{aligned}
$$

Let

$$
\begin{aligned}
A_{j m, k l}= & \left(h_{j}(x) h_{k}(y), h_{m}(x) h_{l}(y)\right)_{\omega} \\
= & \sum_{p=0}^{N} \sum_{q=0}^{N} h_{j}\left(x_{p}\right) h_{m}\left(x_{p}\right) \omega_{p} h_{k}\left(y_{q}\right) h_{n}\left(y_{q}\right) \omega_{q}, \quad 0 \leq j, k, l, m \leq N, \\
B_{j m, k l}= & \left(h_{j}^{\prime}(x) h_{k}(y), h_{m}^{\prime}(x) h_{l}(y)\right)_{\omega}+\left(h_{j}(x) h_{k}^{\prime}(y), h_{m}(x) h_{l}^{\prime}(y)\right)_{\omega} \\
= & \sum_{p=0}^{N} \sum_{q=0}^{N} h_{j}^{\prime}\left(x_{p}\right) h_{m}^{\prime}\left(x_{p}\right) \omega_{p} h_{k}\left(y_{q}\right) h_{l}\left(y_{q}\right) \omega_{q} \\
& +\sum_{p=0}^{N} \sum_{q=0}^{N} h_{j}\left(x_{p}\right) h_{m}\left(x_{p}\right) \omega_{p} h_{k}^{\prime}\left(y_{q}\right) h_{l}^{\prime}\left(y_{q}\right) \omega_{q}, \quad 0 \leq j, k, l, m \leq N,
\end{aligned}
$$

and let

$$
\begin{aligned}
& \boldsymbol{B}=\left(B_{j m, k l}\right)_{(N+1)^{2} \times(N+1)^{2}}, \quad \boldsymbol{A}=\left(A_{j m, k l}\right)_{(N+1)^{2} \times(N+1)^{2}}, \\
& \boldsymbol{U}_{N}^{n}=\left(u_{N_{0,0}}^{n}, u_{N_{1,0}}^{n}, \ldots, u_{N_{N, 0}}^{n}, u_{N_{0,1}}^{n}, u_{N_{1,1}}^{n}, \ldots, u_{N_{N, 1}}^{n}, \ldots, u_{N_{0, N}}^{n}, \ldots, u_{N_{N, N}}^{n}\right)^{T}, \\
& \boldsymbol{F}^{n}=\left(F_{0,0}^{n}, F_{1,0}^{n}, \ldots, n,{ }_{N, 0}, F_{0,1}^{n}, \ldots, F_{N, 1}^{n}, \ldots, F_{0, N}^{n}, \ldots, F_{N, N}^{n}\right)^{T}, \quad F_{m, l}^{n}=f\left(x_{m}, y_{l}, n \Delta t\right) .
\end{aligned}
$$

Therefore, Problem 2 may be rewritten into the following matrix-form. 
Problem 3 Seek $\boldsymbol{U}_{N}^{n} \in \mathbb{R}^{(N+1)^{2}}(n=2,3, \ldots, K)$ such that

$$
\begin{aligned}
& {\left[\left(2+\alpha \Delta t+\beta \Delta t^{2}\right) \boldsymbol{A}+\Delta t^{2} \boldsymbol{B}\right] \boldsymbol{U}_{N}^{n+1}} \\
& \quad=4 \boldsymbol{A} \boldsymbol{U}_{N}^{n}+2 \Delta t^{2} \boldsymbol{F}^{n}-\left[\left(2-\alpha \Delta t+\beta \Delta t^{2}\right) \boldsymbol{A}+\Delta t^{2} \boldsymbol{B}\right] \boldsymbol{U}_{N}^{n-1}, \quad 1 \leq n \leq K-1 .
\end{aligned}
$$

Remark 1 As the coefficient matrixes are constructed by some trigonometric values, in spite that the accuracy of the CCS model is higher than those of other numeric models, for example, the FE, FD, and FVE models, the CCS model is more intricate than other numeric models as it takes more weighty calculating burden. Therefore, the order reduction of the CCS model is more vital than that of other numeric models. Therefore, we take the initial $L$ vectors $\boldsymbol{U}_{N}^{1}, \boldsymbol{U}_{N}^{2}, \ldots, \boldsymbol{U}_{N}^{L}(L \ll K)$ from the set of coefficient vectors $\left\{\boldsymbol{U}_{N}^{n}\right\}_{n=1}^{K}$ for the CCS matrix-format (15) to make up of a $(N+1)^{2} \times L$ snapshot matrix $\boldsymbol{Q}=\left(\boldsymbol{U}_{N}^{1}, \boldsymbol{U}_{N}^{2}, \ldots, \boldsymbol{U}_{N}^{L}\right)$.

\section{The ROECS method for the telegraph equation}

\subsection{Formulation of POD basis}

For the snapshot matrix $\boldsymbol{Q}=\left(\boldsymbol{U}_{N}^{1}, \boldsymbol{U}_{N}^{2}, \ldots, \boldsymbol{U}_{N}^{L}\right)$ constituted in Sect. 2.4, let $\lambda_{1} \geq \lambda_{2} \geq \cdots \geq$ $\lambda_{r}>0(\gamma=: \operatorname{rank}(\boldsymbol{Q}))$ stand for all positive eigenvalues of $\mathbf{Q} \boldsymbol{Q}^{T}$, and let $\boldsymbol{U}=\left(\boldsymbol{\phi}_{1}, \boldsymbol{\phi}_{2}, \ldots, \boldsymbol{\phi}_{r}\right) \in$ $\mathbb{R}^{(N+1)^{2} \times r}$ stand for the eigenmatrix formed by the associated orthonormal eigenvectors of $\mathbf{Q Q}^{T}$. Thus, a set of POD bases $\boldsymbol{\Phi}=\left(\boldsymbol{\phi}_{1}, \boldsymbol{\phi}_{2}, \ldots, \boldsymbol{\phi}_{d}\right)(d \leq \gamma)$ is gained by the first $d$ vectors in $\boldsymbol{U}$ that possess the following property (see $[21,24])$ :

$$
\left\|\boldsymbol{Q}-\boldsymbol{\Phi} \boldsymbol{\Phi}^{T} \mathbf{Q}\right\|_{2,2}=\sqrt{\lambda_{d+1}}
$$

where $\|\mathbf{Q}\|_{2,2}=\sup _{\boldsymbol{v} \neq \mathbf{0}}\|\mathbf{Q} \boldsymbol{v}\|_{2} /\|\boldsymbol{v}\|_{2}$ and $\|\boldsymbol{v}\|_{2}$ stands for the Euclidean norm to vector $\boldsymbol{v}$. It follows that

$$
\begin{aligned}
\left\|\boldsymbol{U}_{N}^{n}-\boldsymbol{\Phi} \boldsymbol{\Phi}^{T} \boldsymbol{U}_{N}^{n}\right\|_{2} & =\left\|\left(\boldsymbol{Q}-\boldsymbol{\Phi} \boldsymbol{\Phi}^{T} \mathbf{Q}\right) \boldsymbol{e}_{n}\right\|_{2} \\
& \leq\left\|\boldsymbol{Q}-\boldsymbol{\Phi} \boldsymbol{\Phi}^{T} \mathbf{Q}\right\|_{2,2}\left\|\boldsymbol{e}_{n}\right\|_{2} \leq \sqrt{\lambda_{d+1}}, \quad 1 \leq n \leq L,
\end{aligned}
$$

where $\boldsymbol{e}_{i}(1 \leq i \leq L)$ stands for the unit vectors whose $i$ th component is 1 .

Remark 2 Thanks to the number of order $L$ of $\mathbf{Q}^{T} \mathbf{Q}$ being far smaller than the number of order $(N+1)^{2}$ of $\mathbf{Q} \mathbf{Q}^{T}$, but both positive eigenvalues $\lambda_{i}(1 \leq i \leq \gamma)$ are identical, one may firstly seek the eigenvalues $\lambda_{i}(1 \leq i \leq \gamma)$ of $\mathbf{Q}^{T} \mathbf{Q}$ and the associated eigenvectors $\boldsymbol{\varphi}_{i}$ $(1 \leq i \leq \gamma)$, and then, according to $\boldsymbol{\phi}_{i}=\boldsymbol{Q} \boldsymbol{\varphi}_{i} / \sqrt{\lambda_{i}}(1 \leq i \leq r)$, one may easily acquire the eigenvectors $\boldsymbol{\phi}_{i}(1 \leq i \leq \gamma)$ of $\mathbf{Q} \mathbf{Q}^{T}$.

\subsection{The ROECS model}

From (17) in Sect. 3.1 one may acquire the initial $L$ coefficient vectors to the ROECS solutions $\boldsymbol{U}_{d}^{n}=\boldsymbol{\Phi} \boldsymbol{\Phi}^{T} \boldsymbol{U}_{N}^{n}=: \boldsymbol{\Phi} \boldsymbol{\beta}_{d}^{n}(n=1,2, \ldots, L \leq K)$, where $\boldsymbol{U}_{d}^{n}=\left(u_{d, 0,0}^{n}, u_{d, 1,0}^{n}, \ldots, u_{d, N, 0}^{n}, u_{d, 0,1}^{n}\right.$, $\left.u_{d, 1,1}^{n}, \ldots, u_{d, N, 1}^{n}, \ldots, u_{d, 0, N}^{n}, u_{d, 1, N}^{n}, \ldots, u_{d, N, N}^{n+1}\right)^{T}$, and $\boldsymbol{\beta}_{d}^{n}=\left(\beta_{1}^{n}, \beta_{2}^{n}, \ldots, \beta_{d}^{n}\right)^{T}$. Thanks to the matrix $\left[\left(2+\alpha \Delta t+\beta \Delta t^{2}\right) \boldsymbol{A}+\Delta t^{2} \boldsymbol{B}\right]$ being reversible, when the coefficient vectors $\boldsymbol{U}_{N}^{n}$ in (15) 
are replaced with $\boldsymbol{U}_{d}^{n}=\boldsymbol{\Phi} \boldsymbol{\beta}_{d}^{n}(L+1 \leq n \leq K)$, one can get the following ROECS model:

$$
\left\{\begin{array}{l}
\boldsymbol{\Phi} \boldsymbol{\beta}_{d}^{n}=\boldsymbol{\Phi} \boldsymbol{\Phi}^{T} \boldsymbol{U}_{N}^{n}, \quad 1 \leq n \leq L \\
\boldsymbol{\Phi} \boldsymbol{\beta}_{d}^{n+1}=4 \mathbb{A} \boldsymbol{A} \boldsymbol{\Phi} \boldsymbol{\beta}_{d}^{n}-\mathbb{A}\left[\left(2-\alpha \Delta t+\beta \Delta t^{2}\right) \boldsymbol{A}+\Delta t^{2} \boldsymbol{B}\right] \boldsymbol{\Phi} \boldsymbol{\beta}_{d}^{n-1}+2 \Delta t^{2} \mathbb{A} \boldsymbol{F}^{n} \\
\quad L \leq n \leq K-1, \\
\boldsymbol{U}_{d}^{n}=\boldsymbol{\Phi} \boldsymbol{\beta}_{d}^{n}, \quad 1 \leq n \leq K
\end{array}\right.
$$

where $\mathbb{A}=\left[\left(2+\alpha \Delta t+\beta \Delta t^{2}\right) \boldsymbol{A}+\Delta t^{2} \boldsymbol{B}\right]^{-1}, \boldsymbol{U}_{N}^{n}(1 \leq n \leq L)$ stand for the initial $L$ coefficient vectors in (15), and $\boldsymbol{A}$ and $\boldsymbol{B}$ are given in (15).

Furthermore, model (18) can be simplified as

$$
\left\{\begin{aligned}
& \boldsymbol{\beta}_{d}^{n}= \boldsymbol{\Phi}^{T} \boldsymbol{U}_{N}^{n}, \quad n=1,2, \ldots, L \\
& \boldsymbol{\beta}_{d}^{n+1}= 4 \boldsymbol{\Phi}^{T} \mathbb{A} \boldsymbol{A} \boldsymbol{\Phi} \boldsymbol{\beta}_{d}^{n}-\boldsymbol{\Phi}^{T} \mathbb{A}\left[\left(2-\alpha \Delta t+\beta \Delta t^{2}\right) \boldsymbol{A}+\Delta t^{2} \boldsymbol{B}\right] \Phi \boldsymbol{\beta}_{d}^{n-1} \\
&+2 \Delta t^{2} \boldsymbol{\Phi}^{T} \mathbb{A} \boldsymbol{F}^{n}, \quad n=L, L+1, \ldots, K-1, \\
& \boldsymbol{U}_{d}^{n}=\boldsymbol{\Phi} \boldsymbol{\beta}_{d}^{n}, \quad n=1,2, \ldots, K .
\end{aligned}\right.
$$

Remark 3 CCS model (15) includes $(N+1)^{2}$ unknowns at every time node, but ROECS model (19) at the same node has only $d$ unknowns (where $d \leq L \ll(N+1)^{2}$, for instance, in the numeric experimentations of Sect. $4,(N+1)^{2}=10201$, whereas $\left.d=6\right)$. It follows that ROECS model (19) is clearly superior to CCS model (15). After having gotten $\boldsymbol{U}_{d}^{n}=\left(u_{d, 0,0}^{n}, u_{d, 1,0}^{n}, \ldots, u_{d, N, 0}^{n}, u_{d, 0,1}^{n}, u_{d, 1,1}^{n}, \ldots, u_{d, N, 1}^{n}, \ldots, u_{d, 0, N}^{n}, u_{d, 1, N}^{n}, \ldots, u_{d, N, N}^{n+1}\right)^{T}$ by (19), we may gain the authentic ROECS solutions $u_{d}^{n}(x, y)=\sum_{j=0}^{N} \sum_{k=0}^{N} u_{d, j, k}^{n} h_{j}(x) h_{k}(y)(1 \leq n \leq K)$.

\subsection{The existence, convergence, and stability of the ROECS solutions}

Analyzing the existence, convergence, and stability of the ROECS solutions requires the following max-norms to matrix as well as vector:

$$
\begin{aligned}
& \|\boldsymbol{D}\|_{\infty}=\max _{1 \leq i \leq m} \sum_{j=1}^{l}\left|d_{i j}\right|, \quad \forall \boldsymbol{D}=\left(d_{i j}\right)_{m \times l} \in \mathbb{R}^{m} \times \mathbb{R}^{l}, \\
& \|\boldsymbol{\chi}\|_{\infty}=\max _{1 \leq j \leq m}\left|\chi_{j}\right|, \quad \forall \boldsymbol{\chi}=\left(\chi_{1}, \chi_{2}, \ldots, \chi_{m}\right)^{T} \in \mathbb{R}^{m} .
\end{aligned}
$$

The existence and stability as well as convergence of the ROECS solutions hold the following consequence.

Theorem 5 Under the same assumptions for Theorem 4, ROECS model (19) has a unique series of solutions $\left\{u_{d}^{n}\right\}_{n=1}^{K}$ satisfying the following stability:

$$
\left\|\nabla u_{d}^{n}\right\|_{0, \omega} \leq C\left(G_{0}, G_{1}, f\right), \quad 1 \leq n \leq K
$$

where $C\left(G_{0}, G_{1}, f\right)$ is the positive constant that only relies on $G_{0}, G_{1}$, and $f$. Furthermore, when $u\left(t_{n}\right) \in H_{\omega}^{m}(\Omega)(2 \leq m \leq N+1)$, the error estimates between the solutions to Problem 1 with the ROECS solutions are as follows:

$$
\left\|u\left(t_{n}\right)-u_{d}^{n}\right\|_{0, \omega} \leq C\left(\Delta t^{2}+N^{-m}+\sqrt{\lambda_{d+1}} N^{-1 / 2} \Delta t^{-1}\right), \quad n=1,2, \ldots, K
$$


Proof (1) The prooffor existence as well as stability of the ROECS solutions.

As $\left[\left(2+\alpha \Delta t+\beta \Delta t^{2}\right) \boldsymbol{A}+\Delta t^{2} \boldsymbol{B}\right]$ is a reversible matrix, from ROECS model (19) as well as Remark 3, we may judge that ROECS model (19) has a unique series of the ROECS solutions.

Using (18), one may restore ROECS model (19) into the following scheme:

$$
\begin{aligned}
& \boldsymbol{U}_{d}^{n}=\boldsymbol{\Phi} \boldsymbol{\Phi}^{T} \boldsymbol{U}_{N}^{n}, \quad 1 \leq n \leq L \\
& \boldsymbol{U}_{d}^{n+1}=4 \mathbb{A} \boldsymbol{A} \boldsymbol{U}_{d}^{n}+2 \Delta t^{2} \mathbb{A} \boldsymbol{F}^{n}-\mathbb{A}\left[\left(2-\alpha \Delta t+\beta \Delta t^{2}\right) \boldsymbol{A}+\Delta t^{2} \boldsymbol{B}\right] \boldsymbol{U}_{d}^{n-1}, \\
& \quad L \leq n \leq K-1 .
\end{aligned}
$$

Note that $\boldsymbol{H}=\left(h_{0}(x) h_{0}(y), h_{1}(x) h_{0}(y), \ldots, h_{N}(x) h_{0}(y), h_{0}(x) h_{1}(y), h_{1}(x) h_{1}(y), \ldots, h_{N}(x) \times\right.$ $\left.h_{1}(y), \ldots, h_{0}(x) h_{N}(y), h_{1}(x) h_{N}(y), \ldots, h_{N}(x) h_{N}(y)\right)^{T}$. Then the solutions to Problem 2 may be denoted by $u_{N}^{n}=\boldsymbol{H}^{T} \boldsymbol{U}_{N}^{n}=\boldsymbol{H} \cdot \boldsymbol{U}_{N}^{n}$. Similarly, $u_{d}^{n}=\boldsymbol{H}^{T} \boldsymbol{U}_{d}^{n}=\boldsymbol{H} \cdot \boldsymbol{U}_{d}^{n}$.

When $1 \leq n \leq L$, we get

$$
\begin{aligned}
\left\|u_{d}^{n}\right\|_{0, \omega} & =\left\|\boldsymbol{\Phi} \boldsymbol{\Phi}^{T} \boldsymbol{H}^{T} \boldsymbol{U}_{N}^{n}\right\|_{0, \omega} \\
& \leq\left\|\boldsymbol{\Phi} \boldsymbol{\Phi}^{T}\right\|_{\infty}\left\|\boldsymbol{H}^{T} \boldsymbol{U}_{N}^{n}\right\|_{0, \omega} \leq\left\|u_{N}^{n}\right\|_{0, \omega}, \quad 1 \leq n \leq L .
\end{aligned}
$$

Thus, uniting Theorem 4 with (24), one can obtain that (20) is right when $1 \leq n \leq L$.

When $L+1 \leq n \leq K$, due to the positive definiteness of the matrix $\boldsymbol{B}$, one can rewrite (23) into

$$
\begin{aligned}
\boldsymbol{B}^{-1} \boldsymbol{A}\left(\boldsymbol{U}_{d}^{n+1}-2 \boldsymbol{U}_{d}^{n}+\boldsymbol{U}_{d}^{n-1}\right)+\frac{\alpha \Delta t \boldsymbol{B}^{-1} \boldsymbol{A}}{2}\left(\boldsymbol{U}_{d}^{n+1}-\boldsymbol{U}_{d}^{n-1}\right) \\
+\frac{\beta \Delta t^{2} \boldsymbol{B}^{-1} \boldsymbol{A}}{2}\left(\boldsymbol{U}_{d}^{n-1}+\boldsymbol{U}_{d}^{n+1}\right)+\frac{\Delta t^{2}}{2}\left(\boldsymbol{U}_{d}^{n-1}+\boldsymbol{U}_{d}^{n+1}\right) \\
=\Delta t^{2} \boldsymbol{B}^{-1} \boldsymbol{F}^{n}, \quad L \leq n \leq K-1 .
\end{aligned}
$$

Moreover, the following inequalities can be attained by the FE theory in [31], the spectral theory in [4], and the matrix properties:

$$
\left\|\boldsymbol{A}^{-1}\right\|_{\infty} \leq C ; \quad\left\|\boldsymbol{B}^{-1}\right\|_{\infty} \leq C N^{-1} ; \quad\|\boldsymbol{A}\|_{\infty} \leq C ; \quad\|\boldsymbol{B}\|_{\infty} \leq C N
$$

Because of the positive definiteness for matrix $\boldsymbol{B}^{-1} \boldsymbol{A}$, there exist an orthogonal matrix $\boldsymbol{B}_{1}$ and a diagonal matrix $\boldsymbol{D}_{1}$ such that

$$
\begin{aligned}
& \left(\boldsymbol{U}_{d}^{n+1}-\boldsymbol{U}_{d}^{n-1}\right)^{T} \boldsymbol{B}^{-1} \boldsymbol{A}\left(\boldsymbol{U}_{d}^{n+1}-\boldsymbol{U}_{d}^{n-1}\right) \\
& =\left(\boldsymbol{U}_{d}^{n+1}-\boldsymbol{U}_{d}^{n-1}\right)^{T}\left(\boldsymbol{B}_{1} \boldsymbol{D}_{1}\right)^{T}\left(\boldsymbol{B}_{1} \boldsymbol{D}_{1}\right)\left(\boldsymbol{U}_{d}^{n+1}-\boldsymbol{U}_{d}^{n-1}\right) \\
& =\left\|\left(\boldsymbol{B}_{1} \boldsymbol{D}_{1}\right)\left(\boldsymbol{U}_{d}^{n+1}-\boldsymbol{U}_{d}^{n-1}\right)\right\|_{2}^{2}, \\
& \left(\boldsymbol{U}_{d}^{n+1}-\boldsymbol{U}_{d}^{n-1}\right)^{T} \boldsymbol{B}^{-1} \boldsymbol{A}\left(\boldsymbol{U}_{d}^{n+1}+\boldsymbol{U}_{d}^{n-1}\right) \\
& =\left(\boldsymbol{U}_{d}^{n+1}-\boldsymbol{U}_{d}^{n-1}\right)^{T}\left(\boldsymbol{B}_{1} \boldsymbol{D}_{1}\right)^{T}\left(\boldsymbol{B}_{1} \boldsymbol{D}_{1}\right)\left(\boldsymbol{U}_{d}^{n+1}-\boldsymbol{U}_{d}^{n-1}\right) \\
& =\left\|\boldsymbol{B}_{1} \boldsymbol{D}_{1} \boldsymbol{U}_{d}^{n+1}\right\|_{2}^{2}-\left\|\boldsymbol{B}_{1} \boldsymbol{D}_{1} \boldsymbol{U}_{d}^{n-1}\right\|_{2}^{2} .
\end{aligned}
$$


Making the scalar product of vector with $\left(\boldsymbol{U}_{d}^{n+1}-\boldsymbol{U}_{d}^{n-1}\right)^{T}$ to (25) and using the CauchySchwarz inequality, (26), and (27), we have

$$
\begin{aligned}
\left(\boldsymbol{U}_{d}^{n+1}-\boldsymbol{U}_{d}^{n}\right)^{T} \boldsymbol{B}^{-1} \boldsymbol{A}\left(\boldsymbol{U}_{d}^{n+1}-\boldsymbol{U}_{d}^{n}\right)-\left(\boldsymbol{U}_{d}^{n}-\boldsymbol{U}_{d}^{n-1}\right)^{T} \boldsymbol{B}^{-1} \boldsymbol{A}\left(\boldsymbol{U}_{d}^{n}-\boldsymbol{U}_{d}^{n-1}\right) \\
\quad+\frac{\alpha \Delta t}{2}\left\|\left(\boldsymbol{B}_{1} \boldsymbol{D}_{1}\right)\left(\boldsymbol{U}_{d}^{n+1}-\boldsymbol{U}_{d}^{n-1}\right)\right\|_{2}^{2}+\frac{\beta \Delta t^{2}}{2}\left(\left\|\boldsymbol{B}_{1} \boldsymbol{D}_{1} \boldsymbol{U}_{d}^{n+1}\right\|_{2}^{2}-\left\|\boldsymbol{B}_{1} \boldsymbol{D}_{1} \boldsymbol{U}_{d}^{n-1}\right\|_{2}^{2}\right) \\
\quad+\frac{\Delta t^{2}}{2}\left(\left\|\boldsymbol{U}_{d}^{n+1}\right\|_{2}^{2}-\left\|\boldsymbol{U}_{d}^{n-1}\right\|_{2}^{2}\right) \\
=\Delta t^{2}\left(\boldsymbol{U}_{d}^{n+1}-\boldsymbol{U}_{d}^{n-1}\right)^{T} \boldsymbol{B}^{-1} \boldsymbol{F}^{n} \\
\leq \frac{\alpha \Delta t}{2}\left\|\left(\boldsymbol{B}_{1} \boldsymbol{D}_{1}\right)\left(\boldsymbol{U}_{d}^{n+1}-\boldsymbol{U}_{d}^{n-1}\right)\right\|_{2}^{2}+C \Delta t^{3} N^{-2}\left\|\boldsymbol{F}^{n}\right\|_{2}^{2}
\end{aligned}
$$

where $n=L, L+1, \ldots, K-1$. Simplifying (29) as well as summating from $L$ through $n$, we have

$$
\begin{aligned}
\left(\boldsymbol{U}_{d}^{n+1}-\boldsymbol{U}_{d}^{n}\right)^{T} \boldsymbol{B}^{-1} \boldsymbol{A}\left(\boldsymbol{U}_{d}^{n+1}-\boldsymbol{U}_{d}^{n}\right)+\frac{\Delta t^{2}}{2}\left(\left\|\boldsymbol{U}_{d}^{n+1}\right\|_{2}^{2}+\left\|\boldsymbol{U}_{d}^{n}\right\|_{2}^{2}\right) \\
\quad+\frac{\beta \Delta t^{2}}{2}\left(\left\|\boldsymbol{B}_{1} \boldsymbol{D}_{1} \boldsymbol{U}_{d}^{n+1}\right\|_{2}^{2}+\left\|\boldsymbol{B}_{1} \boldsymbol{D}_{1} \boldsymbol{U}_{d}^{n}\right\|_{2}^{2}\right) \\
\leq \frac{\Delta t^{2}}{2}\left(\left\|\boldsymbol{U}_{d}^{L}\right\|_{2}^{2}+\left\|\boldsymbol{U}_{d}^{L-1}\right\|_{2}^{2}\right)+\left(\boldsymbol{U}_{d}^{L}-\boldsymbol{U}_{d}^{L-1}\right)^{T} \boldsymbol{B}^{-1} \boldsymbol{A}\left(\boldsymbol{U}_{d}^{L}-\boldsymbol{U}_{d}^{L-1}\right) \\
+\frac{\beta \Delta t^{2}}{2}\left(\left\|\boldsymbol{B}_{1} \boldsymbol{D}_{1} \boldsymbol{U}_{d}^{L}\right\|_{2}^{2}+\left\|\boldsymbol{B}_{1} \boldsymbol{D}_{1} \boldsymbol{U}_{d}^{L-1}\right\|_{2}^{2}\right) \\
+C N^{-2} \Delta t^{3} \sum_{i=L}^{n}\left\|\boldsymbol{F}^{i}\right\|_{2}^{2} \leq \frac{C \Delta t^{2}}{2}\left(\left\|\boldsymbol{U}_{d}^{L}\right\|_{2}^{2}+\left\|\boldsymbol{U}_{d}^{L-1}\right\|_{2}^{2}\right) \\
\quad+C N^{-1}\left\|\boldsymbol{U}_{d}^{L}-\boldsymbol{U}_{d}^{L-1}\right\|_{2}^{2}+C N^{-2} \Delta t^{3} \sum_{i=L}^{n}\left\|\boldsymbol{F}^{i}\right\|_{2}^{2}, \quad L \leq n \leq K-1 .
\end{aligned}
$$

Due to the orthogonality for components of $\boldsymbol{H}$, using the Taylor formula and Theorem 4, we can obtain

$$
\begin{aligned}
\left\|\boldsymbol{U}_{d}^{L}-\boldsymbol{U}_{d}^{L-1}\right\|_{2}= & \left(\left(\boldsymbol{U}_{d}^{L}-\boldsymbol{U}_{d}^{L-1}\right) \boldsymbol{H},\left(\boldsymbol{U}_{d}^{L}-\boldsymbol{U}_{d}^{L-1}\right) \boldsymbol{H}\right)_{\omega}^{1 / 2} \\
= & \left(u_{d}^{L}-u_{d}^{L-1}, u_{d}^{L}-u_{d}^{L-1}\right)_{\omega}^{1 / 2}=\left\|u_{d}^{L}-u_{d}^{L-1}\right\|_{0, \omega} \\
\leq & \left\|u_{d}^{L}-u_{N}^{L}\right\|_{0, \omega}+\left\|u_{N}^{L}-u\left(t_{L}\right)\right\|_{0, \omega}+\left\|u\left(t_{L}\right)-u\left(t_{L-1}\right)\right\|_{0, \omega} \\
& +\left\|u\left(t_{L-1}\right)-u_{N}^{L-1}\right\|_{0, \omega}+\left\|u_{N}^{L-1}-u_{d}^{L}\right\|_{0, \omega} \\
\leq & C\left(G_{0}, G_{1}, f\right)\left(\sqrt{\lambda_{d+1}}+\Delta t+N^{-1}\right) .
\end{aligned}
$$

We derive $\left(\boldsymbol{U}_{d}^{n+1}-\boldsymbol{U}_{d}^{n}\right)^{T} \boldsymbol{B}^{-1} \boldsymbol{A}\left(\boldsymbol{U}_{d}^{n+1}-\boldsymbol{U}_{d}^{n}\right) \geq 0$ from the positive definiteness for $\boldsymbol{B}^{-1} \boldsymbol{A}$. Hence, if $\max \left\{N^{-2}, N^{-1} \lambda_{d+1}\right\} \leq C \Delta t^{2}$ with (31) and (30), we get

$$
\begin{aligned}
\left\|\boldsymbol{U}_{d}^{n+1}\right\|_{2}^{2} & \leq C\left(\left\|\boldsymbol{U}_{d}^{L}\right\|_{2}^{2}+\left\|\boldsymbol{U}_{d}^{L-1}\right\|_{2}^{2}\right)+C\left(G_{0}, G_{1}, f\right)+C N^{-2} \Delta t \sum_{i=L}^{n}\left\|\boldsymbol{F}^{i}\right\|_{2}^{2} \\
& \leq C\left(G_{0}, G_{1}, f\right), \quad n=L, L+1, \ldots, K-1,
\end{aligned}
$$


here $C\left(G_{0}, G_{1}, f\right)$ is the positive constant that only depends on $G_{0}$ and $G_{1}$ as well as $f$. Thus, we have

$$
\left\|u_{d}^{n}\right\|_{0, \omega}=\left\|\boldsymbol{H}(x, y) \cdot \boldsymbol{U}_{d}^{n}\right\|_{0, \omega} \leq C\left(G_{0}, G_{1}, f\right), \quad L=L+1, L+2, \ldots, K,
$$

which signifies that (20) is right when $L+1 \leq n \leq K$.

(2) Estimating errors for the ROECS solutions.

Let $\boldsymbol{E}^{n}=\boldsymbol{U}_{N}^{n}-\boldsymbol{U}_{d}^{n}$. As $n=1,2, \ldots, L$, by (17) we get

$$
\left\|\boldsymbol{E}^{n}\right\|_{2}=\left\|\boldsymbol{U}_{N}^{n}-\boldsymbol{U}_{d}^{n}\right\|_{2}=\left\|\boldsymbol{U}_{N}^{n}-\boldsymbol{\Phi} \boldsymbol{\Phi}^{T} \boldsymbol{U}_{N}^{n}\right\|_{2} \leq \sqrt{\lambda_{d+1}}
$$

When $L+1 \leq n \leq K$, using (15) as well as (25), we have

$$
\begin{aligned}
& \frac{\alpha \Delta t \boldsymbol{B}^{-1} \boldsymbol{A}}{2}\left(\boldsymbol{E}^{n+1}-\boldsymbol{E}^{n-1}\right)+\boldsymbol{B}^{-1} \boldsymbol{A}\left(\boldsymbol{E}^{n-1}-2 \boldsymbol{E}^{n}+\boldsymbol{E}^{n+1}\right) \\
& +\frac{\beta \Delta t^{2} \boldsymbol{B}^{-1} \boldsymbol{A}}{2}\left(\boldsymbol{E}^{n-1}+\boldsymbol{E}^{n+1}\right)+\frac{\Delta t^{2}}{2}\left(\boldsymbol{E}^{n-1}+\boldsymbol{E}^{n+1}\right)=\mathbf{0}, \quad L \leq n \leq K-1 .
\end{aligned}
$$

Making the scalar product of vector with $\left(\boldsymbol{E}^{n+1}-\boldsymbol{E}^{n-1}\right)^{T}$ to (35) and using the CauchySchwarz inequality as well as (26)-(28), we have

$$
\begin{gathered}
\left(\boldsymbol{E}^{n+1}-\boldsymbol{E}^{n}\right)^{T} \boldsymbol{B}^{-1} \boldsymbol{A}\left(\boldsymbol{E}^{n+1}-\boldsymbol{E}^{n}\right)-\left(\boldsymbol{E}^{n}-\boldsymbol{E}^{n-1}\right)^{T} \boldsymbol{B}^{-1} \boldsymbol{A}\left(\boldsymbol{E}^{n}-\boldsymbol{E}^{n-1}\right) \\
+\frac{\alpha \Delta t}{2}\left\|\boldsymbol{B}_{1} \boldsymbol{D}_{1}\left(\boldsymbol{E}^{n+1}-\boldsymbol{E}^{n-1}\right)\right\|_{2}^{2}+\frac{\Delta t^{2}}{2}\left(\left\|\boldsymbol{E}^{n+1}\right\|_{2}^{2}-\left\|\boldsymbol{E}^{n-1}\right\|_{2}^{2}\right) \\
+\frac{\Delta t^{2}}{2}\left(\left\|\boldsymbol{B}_{1} \boldsymbol{D}_{1} \boldsymbol{E}^{n+1}\right\|_{2}^{2}-\left\|\boldsymbol{B}_{1} \boldsymbol{D}_{1} \boldsymbol{E}^{n-1}\right\|_{2}^{2}\right)=0, \quad L \leq n \leq K-1 .
\end{gathered}
$$

Summing (36) from $L$ to $n$ and simplifying it, we have

$$
\begin{aligned}
\left(\boldsymbol{E}^{n+1}-\boldsymbol{E}^{n}\right)^{T} \boldsymbol{B}^{-1} \boldsymbol{A}\left(\boldsymbol{E}^{n+1}-\boldsymbol{E}^{n}\right) \\
\quad+\frac{\Delta t^{2}}{2}\left(\left\|\boldsymbol{E}^{n+1}\right\|_{2}^{2}+\left\|\boldsymbol{E}^{n}\right\|_{2}^{2}+\left\|\boldsymbol{B}_{1} \boldsymbol{D}_{1} \boldsymbol{E}^{n+1}\right\|_{2}^{2}+\left\|\boldsymbol{B}_{1} \boldsymbol{D}_{1} \boldsymbol{E}^{n}\right\|_{2}^{2}\right) \\
\leq \frac{\Delta t^{2}}{2}\left(\left\|\boldsymbol{E}^{L}\right\|_{2}^{2}+\left\|\boldsymbol{E}^{L-1}\right\|_{2}^{2}\right)+\left(\boldsymbol{E}^{L}-\boldsymbol{E}^{L-1}\right)^{T} \boldsymbol{B}^{-1} \boldsymbol{A}\left(\boldsymbol{E}^{L}-\boldsymbol{E}^{L-1}\right) \\
\leq \frac{\Delta t^{2}}{2}\left(\left\|\boldsymbol{E}^{L}\right\|_{2}^{2}+\left\|\boldsymbol{E}^{L-1}\right\|_{2}^{2}\right)+C N^{-1}\left\|\boldsymbol{E}^{L}-\boldsymbol{E}^{L-1}\right\|_{2}^{2} \\
\quad+\frac{\Delta t^{2}}{2}\left(\left\|\boldsymbol{B}_{1} \boldsymbol{D}_{1} \boldsymbol{E}^{L}\right\|_{2}^{2}+\left\|\boldsymbol{B}_{1} \boldsymbol{D}_{1} \boldsymbol{E}^{L-1}\right\|_{2}^{2}\right), \quad n=L, L+1, \ldots, K-1 .
\end{aligned}
$$

Using (34) and (37), we have

$$
\begin{aligned}
\left\|\boldsymbol{E}^{n}\right\|_{2} & \leq C\left(\left\|\boldsymbol{E}^{L}\right\|_{2}+\left\|\boldsymbol{E}^{L-1}\right\|_{2}+N^{-1 / 2} \Delta t^{-1}\left\|\boldsymbol{E}^{L}-\boldsymbol{E}^{L-1}\right\|_{2}\right) \\
& \leq C \sqrt{\lambda_{d+1}} N^{-1 / 2} \Delta t^{-1}, \quad n=L+1, L+2, \ldots, K .
\end{aligned}
$$

Whereupon, using $u_{N}^{n}=\boldsymbol{H} \cdot \boldsymbol{U}_{N}^{n}, u_{d}^{n}=\boldsymbol{H} \cdot \boldsymbol{U}_{d}^{n},\|\boldsymbol{H}\|_{0, \omega} \leq 1$, and the inverse estimation theorem, thanks to the orthogonality for components of $\boldsymbol{H}(x, y)$, we get

$$
\left\|u_{N}^{n}-u_{d}^{n}\right\|_{0, \omega}=\left\|\boldsymbol{H} \cdot \boldsymbol{E}^{n}\right\|_{0, \omega} \leq C \sqrt{\lambda_{d+1}} N^{-1 / 2} \Delta t^{-1}, \quad n=1,2, \ldots, K .
$$


Uniting Theorem 4 with (39) yields (21), and this accomplishes the proof for Theorem 5 .

Remark 4 Theorem 5 is explained in two ways.

(1) The error term $\sqrt{\lambda_{d+1}} N^{-1 / 2} \Delta t^{-1}$ to Theorem 5 is produced from the reduced order procedure for the CCS model, which may be used to suggest the choice for the POD basis, i.e., if only that we opt for $d$ that satisfies $\lambda_{d+1} N^{-1} \Delta t^{-2} \leq \max \left\{\Delta t^{4}, N^{-2 m}\right\}$, we can reach the optimal order error estimates.

(2) Theorem 5 signifies that when the solution $u\left(t_{n}\right) \in H_{\omega}^{m}(\Theta)(3 \leq m \leq N+1)$ to Problem 1, the ROECS solutions relative to the spatial variables possess super-convergence. Even though the solution $u\left(t_{n}\right) \in H_{\omega}^{2}(\Theta)$, the error estimates for the ROECS solutions also reach optimal order, which shows that it is feasible and valid for ROECS model (19) to settle the telegraph equation.

\section{Two sets of numerical experimentations}

Hereby, we propose two sets of numeric experimentations to check out the superiority of the ROECS method for the telegraph equation.

To show the variation in the magnetic field produced by two parallel wires with the same voltage from the top to down, in telegraph equation (1), we take $\alpha=\beta=1$, the computational region $\Omega=(-1,1) \times(-1,1), \Delta t=0.01$, the number of nodes $N=200$ in the $y$ and $x$ directions, $f(x, y, t)=0$, and

$$
\begin{aligned}
& H(x, y)=-H^{0}(x, y) \sqrt{\left|y^{2}-0.25\right|}, \quad(x, y) \in[-1,1] \times[-1,1], \\
& G(x, y)=H^{0}(x, y)|x|, \quad(x, y) \in[-1,1] \times[-1,1],
\end{aligned}
$$

where $H^{0}(x, y)=\frac{1}{36}\left[12+15 \cos \left(\frac{10 r \pi}{3}\right)+6 \cos \left(\frac{10 r \pi}{3}\right)+3 \cos (10 r \pi)\right]$ if $r \leq 0.3$ and $H^{0}(x, y)=0$ when $r>0.3$, and $r=\sqrt{\left|x^{2}-0.25\right|+\left|y^{2}-0.25\right|}$.

We firstly seek out the first 20 solutions $\boldsymbol{U}_{N}^{n}(x)$ with the CCS model, i.e., Problem 2, at initial 20 time nodes $t_{n}(1 \leq n \leq 20)$ forming the snapshot matrix $\boldsymbol{Q}=\left[\boldsymbol{U}_{N}^{1}, \boldsymbol{U}_{N}^{2}, \ldots, \boldsymbol{U}_{N}^{20}\right]$. Next, we seek out the eigenvalues $\lambda_{1} \geq \lambda_{2} \geq \cdots \geq \lambda_{20} \geq 0$ and the corresponding eigenvectors $\varphi_{i}$ $(1 \leq i \leq 20)$ for the matrix $\boldsymbol{Q}^{T} \boldsymbol{Q}$. They are attained by estimating that $\sqrt{\lambda_{d+1}} N^{-1 / 2} \Delta t^{-1} \leq$ $10^{-4}$. Whereupon, we only need to make up the initial six POD bases $\boldsymbol{\Phi}=\left\{\boldsymbol{\phi}_{1}, \boldsymbol{\phi}_{2}, \ldots, \boldsymbol{\phi}_{6}\right\}$ via the formula $\boldsymbol{\phi}_{i}=\boldsymbol{Q} \boldsymbol{\varphi}_{i} / \sqrt{\lambda_{i}}(1 \leq i \leq 6)$. Lastly, the ROECS solutions at $t=1.0$ and 2.0 are found by the ROECS model, as depicted in Figs. 1 and 3, respectively.

To compare reasonability, we also seek out the CCS solutions at $t=1.0$ and 2.0 by the CCS model, i.e., Problem 2, as depicted in Figs. 2 and 4, respectively.

The pair of Figs. 1 and 2 and the pair of Figs. 3 and 4 are nearly the same, but the consequences for the ROECS method are better. Specially, in the aforementioned calculation procedure, the ROECS method at each time node possesses only six degrees of freedom, whereas the CCS model possesses thirty-nine thousand six hundred and one degrees of freedom. By computational records of the CCS model with the ROECS model in the identical Laptop, it has been found that the CPU elapsed time to settle the CCS model on $0 \leq t \leq 2$ takes about five thousand eight hundred seconds, whereas the CPU elapsed time to settle the ROECS model takes about forty-six seconds, namely the CPU elapsed time to the CCS model is about as one hundred and twenty-six times as that to the ROECS model. 


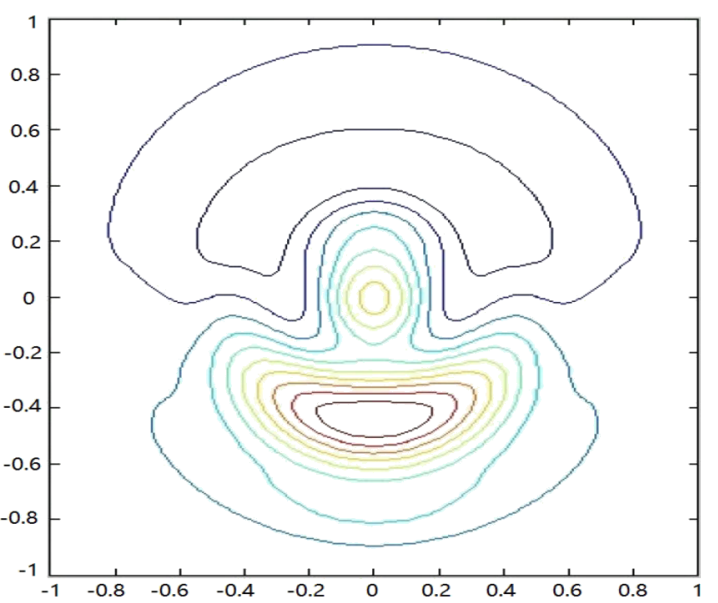

Figure 1 The ROECS solution when $t=1.0$

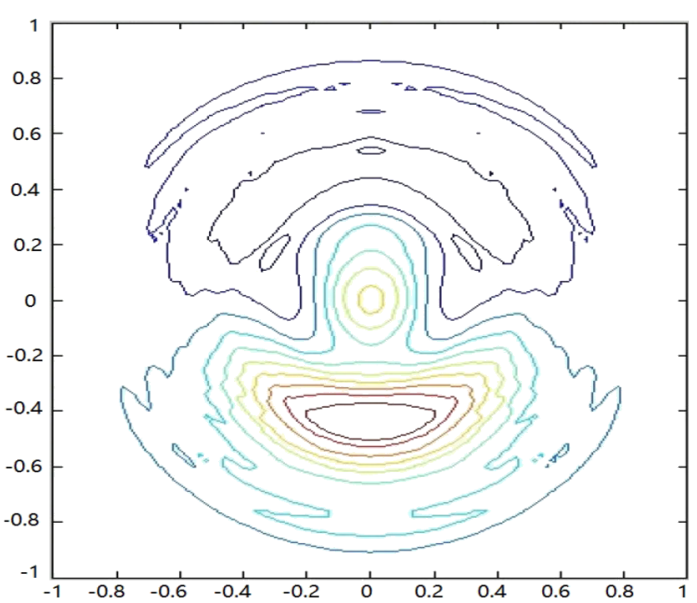

Figure 2 The CCS solution when $t=1.0$

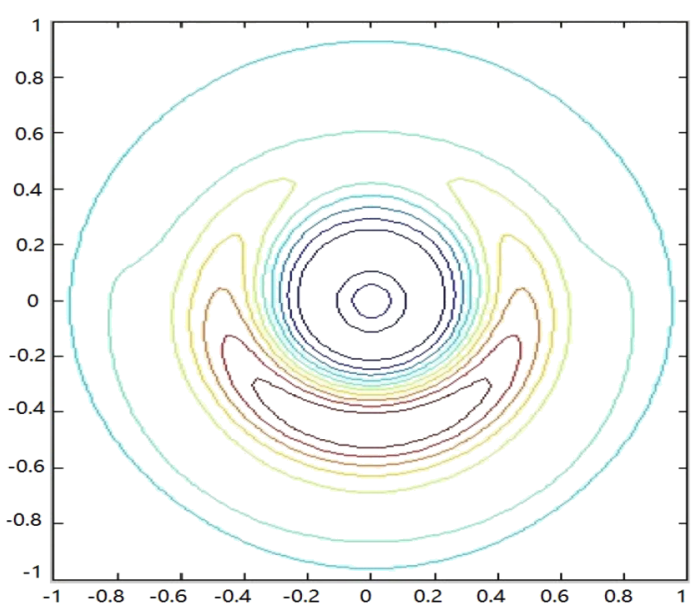

Figure 3 The ROECS solution when $t=2$ 


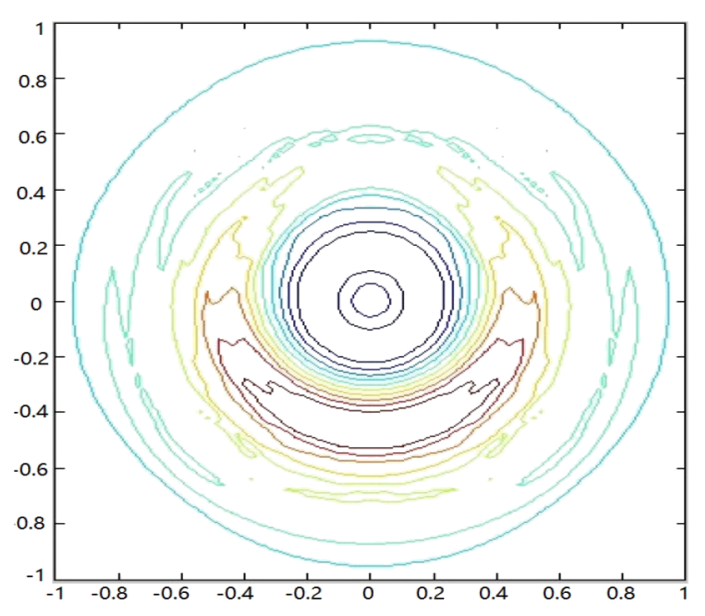

Figure 4 The CCS solution when $t=2.0$

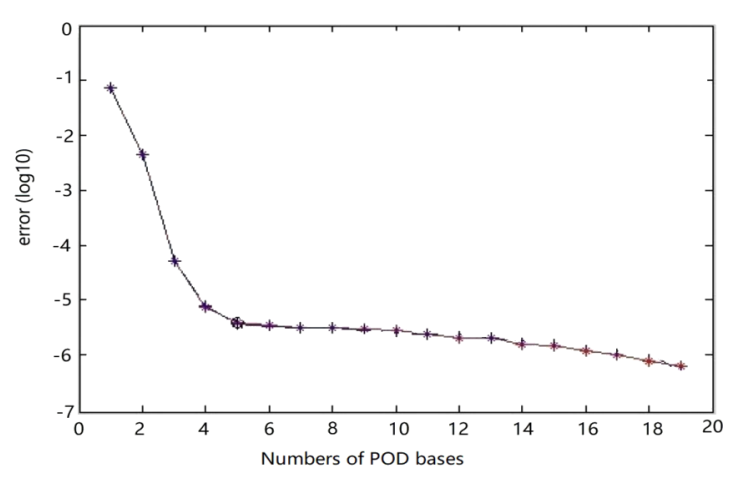

Figure 5 The errors between the ROECS solutions with different number of POD bases and the CCS solution when $t=2.0$

It follows that the ROECS method could not only alleviate the calculation burden as well as decrease the round off error accumulation, but could also greatly spare CPU elapsed time as well as the storage requirements. Figure 5 exhibits the errors between the CCS solution and the ROECS solutions with variational number of POD bases when $t=2.0$, which are consistent with the theory results as both errors reach $O\left(10^{-4}\right)$. This fully validates the correctness of the theory consequences as well as shows that the ROECS model excels far the CCS one.

\section{Conclusions}

Here, the order reduction of the CCS solution coefficient vectors for the telegraph equation has been researched. Based on building the POD-based ROECS model for the telegraph equation, the existence and convergence as well as stability to the ROECS solutions have been proven. Moreover, two sets of numerical experimentations have verified the correctness to the theoretical consequence and illustrated that the ROECS model excels far the CCS one. As the unknowns for ROECS model are far fewer than those for the CCS one, compared with the CCS model, the ROECS model may vastly decrease the calculated burden as well as the accumulation of round off errors and spare the CPU elapsed 
time in the calculations. Most importantly, the ROECS model for the telegraph equation is first proposed and belongs to a fully new development for the existing POD-based reduced order techniques since the accuracy for the ROECS model is far higher than that for other POD reduced order models such as the POD-based reduced order FD, FE, and FVE models.

So far, we have only considered the ROECS model for the telegraph equation in rectangle region $\Omega=(a, b) \times(c, d)$. The approach here may be used to solve the more intricate engineering problems. Hence, it has widespread application prospect in engineering-related field.

\section{Funding}

This research was supported by the National Science Foundation of China Grant 11671106 and Qian Science Cooperation Platform Talent Grant [2017]5726-41.

\section{Availability of data and materials}

The authors declare that all data and material in the paper are available and veritable.

Competing interests

The authors declare that they have no competing interests.

\section{Authors' contributions}

Three authors contributed equally and significantly in writing this article. They wrote, read, and approved the final manuscript.

\section{Author details}

${ }^{1}$ Demonstration Center of Experimental Teaching in Comprehensive Engineering, Beijing Union University, Beijing, China.

${ }^{2}$ School of Mathematics and Physics, North China Electric Power University, Beijing, China.

\section{Publisher's Note}

Springer Nature remains neutral with regard to jurisdictional claims in published maps and institutional affiliations.

Received: 8 October 2019 Accepted: 3 February 2020 Published online: 07 February 2020

\section{References}

1. Hesameddini, E., Asadolahifard, E.: A new spectral Galerkin method for solving the two dimensional hyperbolic telegraph equation. Comput. Math. Appl. 72(7), 1926-1942 (2016)

2. Mittal, R.C., Bhatia, R.: A collocation method for numerical solution of hyperbolic telegraph equation with Neumann boundary conditions. Int. J. Comput. Math. 2014, Article ID 526814 (2014)

3. Luo, Z.D., Jin, S.J.: A reduced-order extrapolated Crank-Nicolson collocation spectral method based on POD for the 2D viscoelastic wave equations. Numer. Methods Partial Differ. Equ. 36, 49-65 (2020)

4. Guo, B.Y.: Spectral Methods and Their Applications. World Scientific, Singapore (1998)

5. Jin, S.J., Luo, Z.D.: A Crank-Nicolson collocation spectral method for the two-dimensional viscoelastic wave equations. Numer. Methods Partial Differ. Equ. 35(3), 1080-1092 (2019)

6. Jin, S.J., Luo, Z.D.: A reduced-order extrapolating collocation spectral method based on POD for the 2D Sobolev equations. Bound. Value Probl. 2019, 63 (2019)

7. Luo, Z.D., Teng, F., Xia, H.: A reduced-order extrapolated Crank-Nicolson finite spectral element method based on POD for the 2D non-stationary Boussinesq equations. J. Math. Anal. Appl. 471(1-2), 564-583 (2019)

8. Luo, Z.D., Jiang, W.R.: The Crank-Nicolson finite spectral element method and numerical simulations for 2D non-stationary Navier-Stokes equations. Math. Methods Appl. Sci. (2019). https://doi.org/10.1002/mma.6039

9. Turkyilmazoglu, M., Cole, J.W., Gajjar, J.S.B.: Absolute and convective instabilities in the compressible boundary layer on a rotating disk. Theor. Comput. Fluid Dyn. 14(1), 21-37 (2000)

10. Turkyilmazoglu, M.: Numerical computation of unsteady flows with an implicit spectral method over a disk in still air. Numer. Heat Transf., Part A, Appl. 57(1), 40-53 (2010)

11. Turkyilmazoglu, M.: Unsteady MHD flow with variable viscosity: applications of spectral scheme. Int. J. Therm. Sci. 49(3), 563-570 (2010)

12. Elgindy, K.T.: Higher-order numerical solution of second-order one-dimensional hyperbolic telegraph equation using a shifted Gegenbauer pseudospectral method. Numer. Methods Partial Differ. Equ. 32(1), 307-349 (2016)

13. Hashemi, M.S., Baleanu, D.: Numerical approximation of higher-order time-fractional telegraph equation by using a combination of a geometric approach and method of line. J. Comput. Phys. 316, 10-20 (2016)

14. He, S., Li, H.: Time discontinunous space-time finite element method for telegraph equations. Appl. Math. J. Chin. Univ. Ser. A 27(4), 425-438 (2012)

15. Jiwari, R., Pandit, S., Mittal, R.C.: A differential quadrature algorithm to solve the two dimensional linear hyperbolic telegraph equation with Dirichlet and Neumann boundary conditions. Appl. Math. Comput. 218, 7279-7294 (2012)

16. Ma, W.T., Zhang, B.W., Ma, H.L.: A meshless collocation approach with barycentric rational interpolation for two-dimensional hyperbolic telegraph equation. Appl. Math. Comput. 279, 236-248 (2016) 
17. Mohanty, R.K.: New unconditionally stable difference schemes for the solution of multi-dimensional telegraphic equations. Int. J. Comput. Math. 86(12), 2061-2071 (2009)

18. Zhou, Y.J., Luo, Z.D.: A Crank-Nicolson collocation spectral method for the two-dimensional telegraph equations. J. Inequal. Appl. 2018, 137 (2018)

19. Kunisch, K., Volkwein, S.: Galerkin proper orthogonal decomposition methods for parabolic problems. Numer. Math. 90(1), 117-148 (2001)

20. Teng, F., Luo, Z.D.: A reduced-order extrapolation technique for solution coefficient vectors in the mixed finite element method for the 2D nonlinear Rosenau equation. J. Math. Anal. Appl. 485(1), 123761 (2020)

21. Luo, Z.D., Chen, G.: Proper Orthogonal Decomposition Methods for Partial Differential Equations. Academic Press, San Diego (2018)

22. Luo, Z.D., Jiang, W.R.: A reduced-order extrapolated finite spectral element method for the 2D non-stationary Navier-Stokes equations about vorticity-stream functions. Appl. Numer. Math. 147, 161-173 (2020)

23. Yang, J., Luo, Z.D.: A POD reduced-order extrapolation continuous space-time finite element method for the $2 \mathrm{D}$ unsteady Stokes equation. J. Math. Anal. Appl. 3475, 123-138 (2019)

24. Luo, Z.D., Wang, H.: A highly efficient reduced-order extrapolated finite difference algorithm for time-space tempered fractional diffusion-wave equation. Appl. Math. Lett. 102, 106090 (2020)

25. Teng, F., Luo, Z.D., Yang, J.: A reduced order extrapolated natural boundary element method based on POD for the 2D hyperbolic equation in unbounded domain. Math. Methods Appl. Sci. 42, 4273-4291 (2019)

26. Luo, Z.D., Xie, Z.H., Shang, Y.Q., Chen, J.: A reduced finite volume element formulation and numerical simulations based on POD for parabolic problems. J. Comput. Appl. Math. 235(8), 2098-2111 (2011)

27. Teng, F., Luo, Z.D., Yang, J.: A reduced order extrapolated NBE format based on POD for the 2D parabolic equation in unbounded domain. Comput. Appl. Math. 38(3), 102 (2019)

28. Hesthaven, J.S., Rozza, G., Stamm, B.: Certified Reduced Basis Methods for Parametrized Partial Differential Equations. Springer, Cham (2016)

29. Quarteroni, A., Manzoni, A., Negri, F.: Reduced Basis Methods for Partial Differential Equations. Springer, Cham (2016)

30. Adams, R.A.: Sobolev Spaces. Academic Press, New York (1975)

31. Luo, Z.D.: The Foundations and Applications of Mixed Finite Element Methods. Science Press, Beijing (2006) (in Chinese)

\section{Submit your manuscript to a SpringerOpen ${ }^{\circ}$ journal and benefit from:}

- Convenient online submission

- Rigorous peer review

Open access: articles freely available online

- High visibility within the field

- Retaining the copyright to your article

Submit your next manuscript at $\boldsymbol{~ s p r i n g e r o p e n . c o m ~}$ 